\title{
A Numerical Analysis of Nonlinear Waves Generated by Ships of Arbitrary Waterline
}

\author{
(Second Report) \\ by Hideaki Miyata*, Member Kiyohira Aoki**, Member \\ Hisashi Kajitani*, Member
}

\begin{abstract}
Summary
The accuracy of the finite-difference method TUMMAC-II developed in the first report is attempted to be raised by applying the concept of two-step Lax-Wendroff scheme to the computation of convective terms of the momentum equations. The advantages and disadvantages are studied through test computations. The wave configuration and wave resistance of the forebodies of two simple hull forms, with a sharp and a blunt bow, are computed at several speed of advance and the availability of the TUMMAC-II method to hull form design is discussed.
\end{abstract}

\section{Introduction}

In the first report the TUMMAC-II method was developed by extending the treatment of arbitrary body configuration to three-dimensions. This enabled us to compute waves of forebodies of ships of arbitrary waterline with vertical walls. The boundary conditions were carefully examined, aiming at the improvement of the degree of accuracy. Numerical tests showed that the TUMMAC-II method could explain the qualitative characteristics of the nonlinear waves called free surface shock wave (FSSW). However, the quantitative accuracy was not satisfactory.

The accuracy can be easily raised by using finer cells, but it is not adequately admitted from economical standpoint and the limited storage of computer. From these reasons, the accuracy is attempted to be raised by improving the differencing method. The concept of the two-step LaxWendroff method is employed in this second report.

The principal purpose of the numerical analysis of nonlinear waves generated by ships is to develop a reliable method of evaluating wave resistance, taking into account the occurrence of FSSW. The relative accuracy of the evaluated wave resistance can determine the hull form of

* Department of Naval Architecture, The University of Tokyo

** Kawasaki Heavy Industries, Co. (Graduate School, The University of Tokyo during this work) least wave resistance. The present numerical method, based on direct computation of NavierStokes equations under the exact nonlinear free surface conditions, is especially useful to hull forms whose nonlinear component of wave resistance plays a significant role. Some part of the labors presently devoted to model tank tests may be reduced by the new computational tool in future. It will be promoted by the progress of high-speed computer. In this report, a preliminary study is made on the variation of computed wave configuration due to the difference of hull forms and on the evaluation of wave resistance by integrating the computed pressure on hull surfaces. Two hull forms of vertical side-walls with a sharp or blunt bow are chosen for this purpose.

The variables are as defined in the nomenclature of the first report, though some other additional variables are defined, when necessary.

\section{Development of the TUMMAC-IIC method}

\subsection{Two-step Lax-Wendroff method}

The Lax-Wendroff method and its modifications are well known to be useful to the computation of shock waves in supersonic flow. They generally possess high accuracy and adequate stability. The phenomenon of FSSW, having a steep discontinuous forward face of waves, is very similar to shock waves in supersonic flow in many respects. Thus the concept of the two-step Lax-Wendroff method is applied to the TUMMAC-II method and this new version is called TUMMAC-IIC. 
The Lax-Wendroff (LW) method is briefly described below through a one-dimensional convection equation. Suppose the differential equation is,

$$
\partial V / \partial t+\partial E / \partial x=0
$$

where

$$
V \equiv V(x, t), \quad E \equiv E(V)
$$

Eq. (1) is deformed as,

$$
\partial V / \partial t+A \cdot \partial V / \partial x=0
$$

where

$$
A \equiv \partial E / \partial V
$$

By Taylor expansion,

$$
\begin{aligned}
V(x, t+\Delta t)= & V(x, t)+\Delta t \cdot \partial V / \partial t \\
& +\Delta t^{2} / 2 \cdot \partial^{2} V / \partial t^{2}+0\left(\Delta t^{3}\right)
\end{aligned}
$$

In this equation $t$ and $\Delta t$ are time and its increment, respectively. The first and the second derivatives in time of Eq. (3) can be expressed by the derivatives in space by the following relations, and then an explicit expression of the value at the next time step is derived.

$$
\begin{aligned}
& \partial V / \partial t=-\partial E / \partial x \\
& \partial^{2} V / \partial t^{2}=\partial / \partial x(A \cdot \partial E / \partial x)
\end{aligned}
$$

By centered differencing in space, Eq. (3) is represented in a finite-difference form as,

$$
\begin{aligned}
V_{i}^{n+1}= & V_{i}^{n}-D T \cdot\left(E_{i+1}^{n}-E_{i-1}^{n}\right) /(2 \cdot D X) \\
& +\left(D T^{2} / 2\right)\left\{A_{i+1 / 2}\left(E_{i+1}^{n}-E_{i}^{n}\right) / D X\right. \\
& \left.-A_{i-1 / 2}\left(E_{i}^{n}-E_{i-1}^{n}\right) / D X\right\} / D X
\end{aligned}
$$

As the centered differencing gives second-order accuracy in space, the above differencing gives second-order accuracy in both space and time. The previous versions of TUMMAC-II do not give second-order accuracy in time but only firstorder one, and then this kind of differencing may improve the degree of accuracy.

Some modifications of the LW-method are proposed partly in order to reduce computational time. One well-known is the two-step LWmethod $^{23)}$ that eliminates the matrix computation of $A$ in Eq. (2) in multi-dimensional case. A version of the two-step LW-method by Rubin et $a ._{.}{ }^{2,3)}$ is described for the one-dimensional case of Eq. (1) by the following two equations that correspond to Eq. (5) of the LW-method.

$$
\begin{aligned}
\overline{V_{i+1 / 2}^{n+1}}= & 1 / 2\left(V_{i+1}^{n}+V_{i}^{n}\right)-D T\left(E_{i+1}^{n}-E_{i}^{n}\right) / D X \\
V_{i}^{n+1}= & V_{i}^{n}-D T \cdot 1 / 2\left\{\left(E_{i+1}^{n}-E_{i-1}^{n}\right) /(2 \cdot D X)\right. \\
& \left.+\left(\overline{E_{i+1 / 2}^{n+1}}-\overline{E_{i-1 / 2}^{n+1}}\right) / D X\right\}
\end{aligned}
$$

The value $V$ is first evaluated at $i+1 / 2$ in the first-step equation (6a) and its space derivative is of centered differencing with the spacing of $D X / 2$. The value $V$ with a bar obtained from
Eq. (6a) is an intermediate value and is used in the computation of the last term of the secondstep equation (6b). The mean of the two spacedifferencing is taken in Eq. (6b). This two-step scheme is equivalent to the single-step scheme of Eq. (5), when $E$ is a linear function of $V$, and is superior to other methods ${ }^{2)}$ from the standpoint of stability.

\subsection{Application of the two-step Lax-Wendroff method to TUMMAC-II}

The basic differential equation is in threedimensions,

$$
\partial V / \partial t+\partial E / \partial x+\partial F / \partial y+\partial G / \partial z=0
$$

$E, F$ and $G$ are functions of the basic variable $V$. For compressible flow the three equations of mass, momentum and energy are put in order and expressed by Eq. (7). And then, the variables, i.e., velocities, density and pressure, are simultaneously obtained by the difference equation of Eq. (7) by means of the two-step LW-method.

However, the circumstances are quite different for the TUMMAC-II method for incompressible flow. In its computational algorithm velocities and pressure are computed through independent procedures. Therefore, the direct application of the two-step LW-method is not possible. Hence, the concept of the method is applied to the computation of only the convection terms of the Navier-Stokes equations so that the basic algorithm of the TUMMAC-II method is not distorted.

The Navier-Stokes equations are written as,

$$
\begin{gathered}
\partial V / \partial t+\partial E / \partial x+\partial F / \partial y+\partial G / \partial z \\
\quad=-\operatorname{grad} \psi+\nu \cdot \Delta V+B
\end{gathered}
$$

where

$$
\begin{aligned}
& V \equiv\left(\begin{array}{c}
u \\
v \\
w
\end{array}\right), E \equiv\left(\begin{array}{c}
u^{2} \\
v u \\
w u
\end{array}\right), F \equiv\left(\begin{array}{c}
u v \\
v^{2} \\
w v
\end{array}\right), \\
& G \equiv\left(\begin{array}{c}
u w \\
v w \\
w^{2}
\end{array}\right), B \equiv\left(\begin{array}{l}
0 \\
0 \\
g
\end{array}\right)
\end{aligned}
$$

The LHS is the convective terms to be approximated by the two-step LW-method as described below.

For simplicity, a 1-D Navier-Stokes equation is used instead of Eq. (8) for the explanation of the application, as follows.

$$
\partial u / \partial t+\partial\left(u^{2}\right) / \partial x=-Q+S
$$

where

$$
Q \equiv \partial \psi / \partial x, \quad S \equiv \nu \cdot \partial^{2} u / \partial x^{2}
$$

The presence of of the RHS is different from Eq. (1) or (7), and they are considered in the finitedifference representation based on the concept of the two-step LW-method, following Eqs. (6a) and (6b) as, 


$$
\begin{aligned}
& \overline{u_{i+1 / 2}^{n+1}}=1 / 2\left(u_{i}^{n}+u_{i+1}^{n}\right)-D T\left\{\left(u^{2}\right)_{i+1}^{n}-\left(u^{2}\right)_{i}^{n}\right\} / D X \\
& -D T\left\{Q_{i+1 / 2}-S_{i+1 / 2}\right\} \\
& u_{i}^{n+1}=u_{i}^{n}-D T \cdot 1 / 2\left[\left\{\left(u^{2}\right)_{i+1}^{n}-\left(u^{2}\right)_{i-1}^{n}\right\} /(2 \cdot D X)\right. \\
& \left.+\left\{\overline{\left(u^{2}\right)_{i+1 / 2}^{n+1}}-\overline{\left(u^{2}\right)_{i-1 / 2}^{n+1}}\right\} / D X\right] \\
& -D T\left\{Q_{i}-S_{i}\right\}
\end{aligned}
$$

The convective term in a finite-difference form is denoted $U C_{i}{ }^{n}$ and then the Navier-Stokes equation writes as,

$$
u_{i}^{n+1}=u_{i}^{n}-D T \cdot U C_{i}^{n}+D T \cdot S_{i}-D T \cdot Q_{i}
$$

From Eqs. (10b) and (11),

$$
\begin{aligned}
& U C_{i}^{n}=1 / 2\left[\left\{\left(u^{2}\right)_{i+1}^{n}-\left(u^{2}\right)_{i-1}^{n}\right\} /(2 \cdot D X)\right. \\
& \left.\left\{\overline{\left(u^{2}\right)_{i+1 / 2}^{n+1}}-\overline{\left(u^{2}\right)_{i-1 / 2}^{n+1}}\right\} / D X\right]
\end{aligned}
$$

A new finite-difference representation of the convective term is derived from the manipulation based on the two-step LW-method.

In the present 3-D case of TUMMAC-II the finite-difference representations of convective terms are much more complicated. And besides, the staggered mesh system employed in the TUMMAC-II method is not suited to this kind of expression and the manipulation requires additional efforts.

For the explanation of the concrete manipulation in the present 3-D case, assume a summation notation defined in 2-D case as,

$$
\left.\begin{array}{l}
\sum a_{i+1}^{i} j_{j+1}^{j} \equiv a_{i j}+a_{i j+1}+a_{i+1 j}+a_{i+1}{ }_{j+1} \\
\sum a_{i j+1 / 2 \pm 1 / 2} \equiv a_{i}{ }_{j+1}^{j} \equiv a_{i j}+a_{i j+1}
\end{array}\right\}
$$

The first-step equation that corresponds to Eq. (10a) is in the $3-D$ case,

$$
\begin{aligned}
\overline{u_{i j-1 / 2}^{n+1} \cdot k-1 / 2} & =u_{i j-1 / 2 \cdot k-1 / 2} \\
& -D T\left\{\frac{\partial\left(u^{2}\right)}{\partial x}+\frac{\partial(v u)}{\partial y}+\frac{\partial(w u)}{\partial z}\right\}_{i j-1 / 2 \cdot k-1 / 2} \\
& -D T\left\{\frac{\partial \psi}{\partial x}-\nu\left(\frac{\partial^{2} u}{\partial x^{2}}+\frac{\partial^{2} u}{\partial y^{2}}+\frac{\partial^{2} u}{\partial z^{2}}\right)\right\}_{i j-1 / 2 \cdot k-1 / 2}
\end{aligned}
$$

The two terms of convection is, for example, evaluated by centered differencing with the spacing of $D X / 2$ etc. as, (see Fig. 1)

$$
\begin{aligned}
& \left\{\frac{\partial\left(u^{2}\right)}{\partial x}\right\}_{i j-1 / 2 \cdot k-1 / 2} \\
& \quad=\frac{1}{D X}\left\{\left(u_{i+1 / 2 \cdot j-1 / 2 \cdot k-1 / 2}\right)^{2}-\left(u_{i-1 / 2 \cdot j-1 / 2 \cdot k-1 / 2}\right)^{2}\right\}
\end{aligned}
$$

where

$$
\left.\begin{array}{l}
u_{i+1 / 2 \cdot j-1 / 2 \cdot k-1 / 2}=\frac{1}{4} \sum u_{i+1 / 2 \cdot j-1 / 2 \pm 1 / 2 \cdot k-1 / 2 \pm 1 / 2} \\
u_{i-1 / 2 \cdot j-1 / 2 \cdot k-1 / 2}=\frac{1}{4} \sum u_{i-1 / 2 \cdot j-1 / 2 \pm 1 / 2 \cdot k-1 / 2 \pm 1 / 2}
\end{array}\right\}
$$

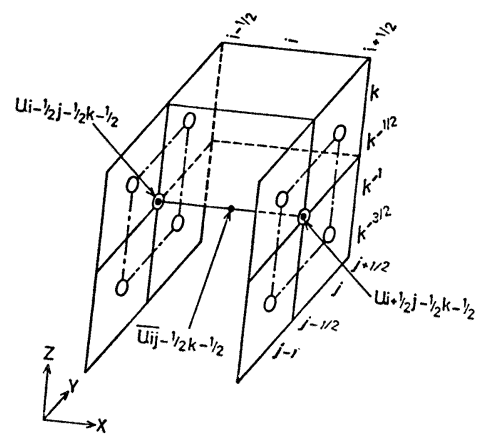

Fig. 1 Velocity points for the first-step

$$
\begin{aligned}
& \left\{\frac{\partial(v u)}{\partial y}\right\}_{i j-1 / 2 \cdot k-1 / 2} \\
& \quad=\frac{1}{D Y}\left\{(v u)_{i j k-1 / 2}-(v u)_{i j-1 k-1 / 2}\right\}
\end{aligned}
$$

where

$$
\begin{aligned}
(v u)_{i j k-1 / 2}= & v_{i j k-1 / 2} \cdot u_{i j k-1 / 2} \\
= & \left(\frac{1}{4} \sum v_{i j \pm 1 / 2 \cdot k-1 / 2 \pm 1 / 2}\right) \\
& \cdot\left(\frac{1}{4} \sum u_{i \pm 1 / 2 \cdot j k-1 / 2 \pm 1 / 2}\right)
\end{aligned}
$$

Next, at the second-step the $x$-directional convective term is calculated, following Eq. (12) as,

$$
\begin{aligned}
U C_{i-1 / 2 \cdot j k}^{n}= & \frac{1}{2}\left[\left(\frac{\partial\left(u^{2}\right)}{\partial x}+\frac{\partial(v u)}{\partial y}+\frac{\partial(w u)}{\partial z}\right)_{i-1 / 2 \cdot j k}^{n}\right. \\
& +\left(\frac{\partial\left(u^{2}\right)}{\partial x}+\frac{\partial(v u)}{\partial y}+\frac{\partial(w u)}{\partial z}\right)_{i-1 / 2 \cdot j k}^{n+1}
\end{aligned}
$$

The former terms without bar in RHS of Eq. (19) is evaluated in the same manner with Eqs. (15) and (17) but with the spacing of $D X$ etc. for the centered differencing. The latter terms are evaluated from the values obtained at the firststep in the following way. (see Fig. 2)

$$
\overline{\left(\frac{\partial\left(u^{2}\right)}{\partial x}\right\}_{i-1 / 2 \cdot j k}^{n+1}}=\frac{1}{D X}\left[\left\{\overline{\left.u_{i j k}^{n+1}\right\}^{2}}-\overline{\left\{u_{i-1 j k}^{n+1}\right\}^{2}}\right]\right.
$$

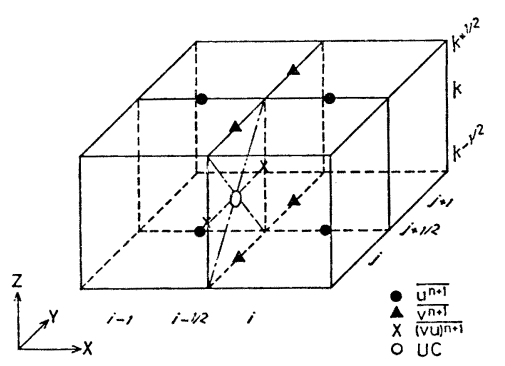

Fig. 2 Velocity points for the second-step 
where

$$
\begin{aligned}
& \overline{u_{i j k}^{n+1}}=\frac{1}{4} \sum \overline{u_{i j \pm 1 / 2}^{n+1} \cdot k \pm 1 / 2} \\
& \overline{\left\{\frac{\partial(v u)}{\partial y}\right\}_{i-1 / 2 \cdot j k}^{n+1}} \\
& =\frac{1}{D Y}\left[\overline{(v u)_{i-1 / 2}^{n+1} \cdot j+1 / 2 \cdot k}-\overline{(v u)_{i-1 / 2 \cdot j-1 / 2 \cdot k}^{n+1}}\right]
\end{aligned}
$$

where

$$
\begin{aligned}
\overline{(v u)_{i-1 / 2}^{n+1} \cdot j+1 / 2 \cdot k}= & \overline{v_{i-1 / 2}^{n+1} \cdot j+1 / 2 \cdot k} \overline{u_{i-1 / 2}^{n+1} \cdot j+1 / 2 \cdot k} \\
= & \left(\frac{1}{4} \sum \overline{v_{i-1 / 2}^{n+1} \cdot j+1 / 2 \pm 1 / 2 \cdot k \pm 1 / 2}\right) \\
& \cdot\left(\frac{1}{4} \sum \overline{u_{i-1 / 2 \pm 1 / 2}^{n+1} \cdot j+1 / 2 \cdot k \pm 1 / 2}\right)
\end{aligned}
$$

From the complicated manipulations abovedescribed the convective terms of the NavierStokes equations are approximated in the manner of the two-step LW-method, and they are introduced in the computational scheme of the TUMMAC-II method. The new version is called TUMMAC-IIC.

\subsection{Boundary conditions}

All the boundary conditions employed in TUMMAC-IIBC are used for TUMMAC-IIC except the interpolation formula in the free surface condition. The four-points interpolation is used in TUMMAC-IIC, because the nine-points interpolation based on a quadratic equation violates the stability of waves. It is presumably because the convection terms work very sensitively near the free surface. The filtering of wave configuration is made in the same way with TUMMAC-IIBc.

\section{Numerical test of TUMMAC-IIC}

\subsection{Conditions of computation}

The wedge model of $\alpha=20^{\circ}$ and a simplified hull form M53A are chosen for numerical test. The conditions of computation are exactly same with those in the first report. The parameters $D X, D Y, D Z, D T$ are $36.0,13.1,25.0 \mathrm{~mm}$ and $0.00459 \mathrm{sec}$. for a wedge and 50.0, 20.0, $25.0 \mathrm{~mm}$ and $0.00252 \mathrm{sec}$. for M53A.

An artificial viscosity is introduced and $R n$ is assumed 100 after the second-step to calculate $\xi, \eta$ and $\zeta$, but the kinematic viscosity at the first step is assumed zero.

\subsection{Computed results and discussion}

The computed wave contour map of a wedge by TUMMAC-IIC is compared with one by TUMMAC-IIBc in Fig. 3. The interval of the contours is $0.1 \mathrm{H}$. The higher wave contours imply that the accuracy is raised by IIC in spite of the expected numerical dissipation of wave height due to the
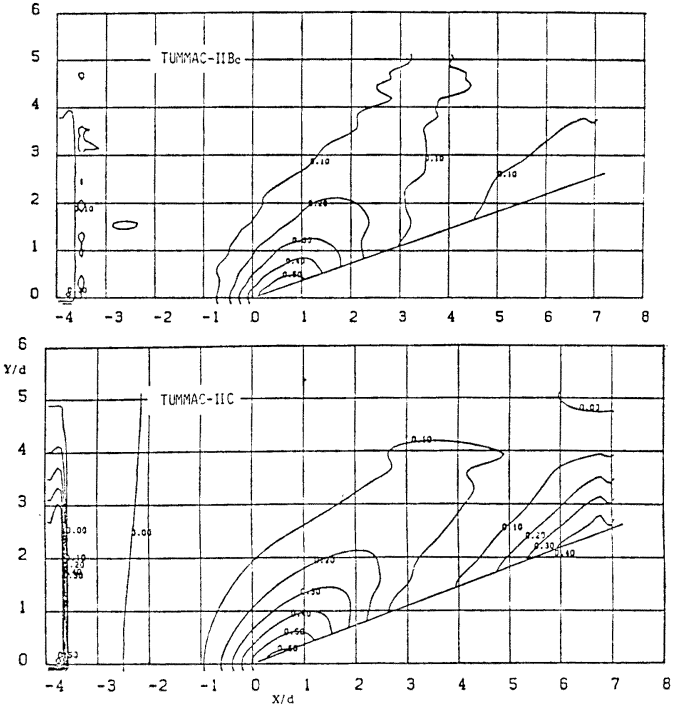

Fig. 3 Wave height contours of a wedge of $\alpha=20^{\circ}$ computed by TUMMACIIBc at $N=480$ (above) and TUMMAC-IIC at $N=540$ (below), $F_{d}=1.1$
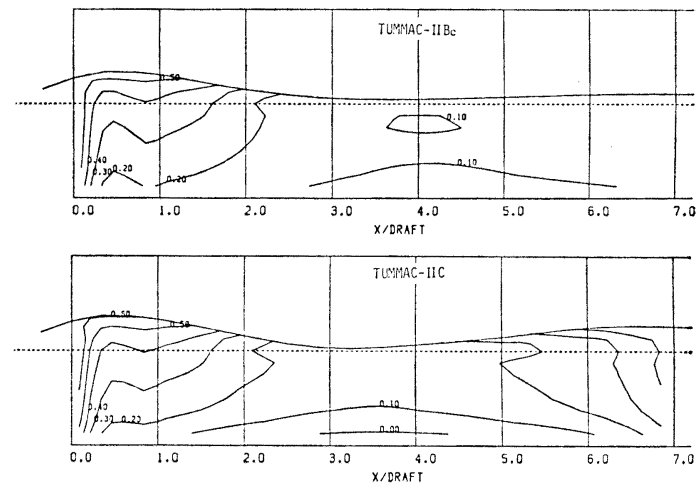

Fig. 4 Computed contours of pressure coefficient at the conditions of Fig. 3, TUMMAC-IIBc (above) and TUMMAC-IIC (below)

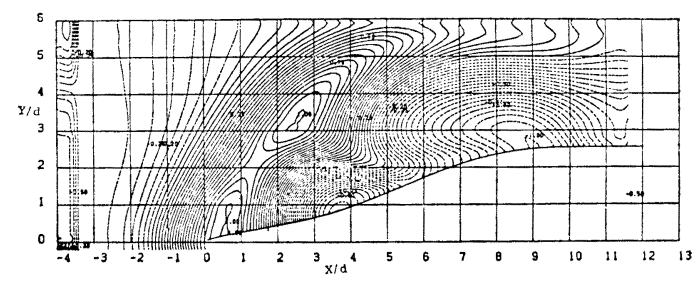

Fig. 5 Wave height contours of M53A at $F_{n}=0.18$ computed by TUMMACIIC, $N=480$ 
four-points interpolation. The remarkable appearance of the second FSSW is most impressive and this is supposed to display the favorable property of IIC which is advantageous to steep or discontinuous wave phenomena.

The computational time required by IIC is about two-times greater than IIBc, which is mostly attributed to a little tedious manipulation for the computation of convective terms described in Chapter 2.

The contour maps of pressure coefficient on the side wall of a wedge computed by TUMMAC-IIBc and IIC are present in Fig. 4. Higher pressure is made conspicuous by IIC at the fore-end and around the second wave. The condition of the fore-end is improved in comparison to TUMMAC-I as described in the first report, and consequently the intimate contours near the fore-end is much more realistic. For most of hull forms the pressure around the stagnant point is extremely important for the computation of resistance by integrating pressure, and then, this is advantageous for practical purposes.

When TUMMAC-IIC is applied to M53A, the computed wave contour map becomes quite strange, as seen in Fig. 5. The difference between a wedge and M53A is only in the configuration of waterlines, that is, the diagonal of a cell is a boundary segment in the case of a wedge, whereas the segment is quite arbitrary in the case of M53A. The arbitrary boundary condition on the body surface as well as the treatment of the cell that contains both body boundary and free surface are presumably the causes of the strange waves. It will be concluded that the TUMMAC-IIC method is very sensitive to boundary conditions and it is not yet suited to practical applications, unless further improvement is made.

\section{Wave resistance computation of ship forebodies}

\subsection{Two simple hull forms and free surface} shock wave

Two simple hull forms with vertical walls WM-1C and WM-1B are used for the computation. These are the first two models used in the experiment on $\mathrm{FSSW}^{4)}$. WM-1C has a simple parabolic waterline and attaching to it a vertical blunt bow whose radius is $40 \mathrm{~mm}$ makes WM-1B. The length, breadth and draft are $2.40 \mathrm{~m}, 0.24 \mathrm{~m}$ and $0.10 \mathrm{~m}$, respectively, being common to the two.

The appearance of waves and experimental wave resistance curves are present in Figs. 6 to 8. The condition of draft in Fig. 6 is not $0.10 \mathrm{~m}$ but $0.06 \mathrm{~m}$, and is not the condition of computation. But the difference of characteristics between the two models is not influenced at all. The fine
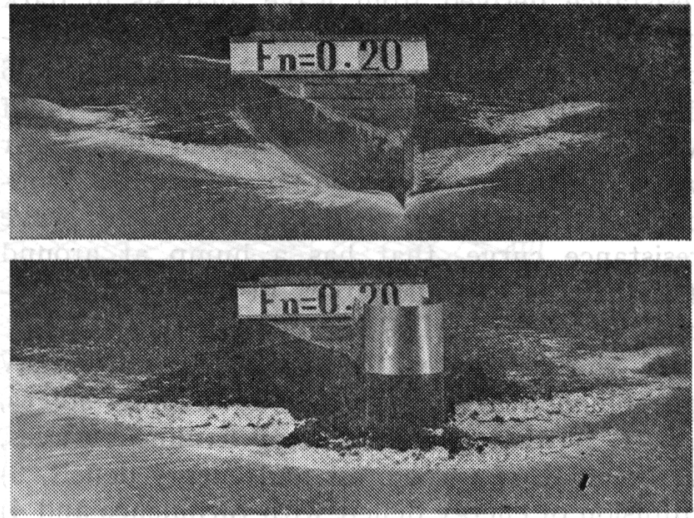

Fig. 6 Bow waves of WM-1C (above) and WM-1B (below) at $F_{n}=0.20, d=$ $0.06 \mathrm{~m}$

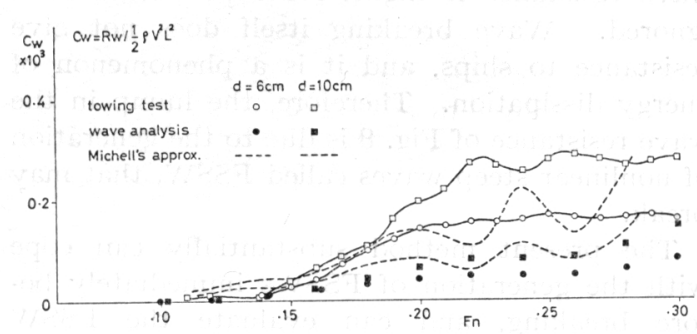

Fig. 7 Wave resistance curves of WM-1C

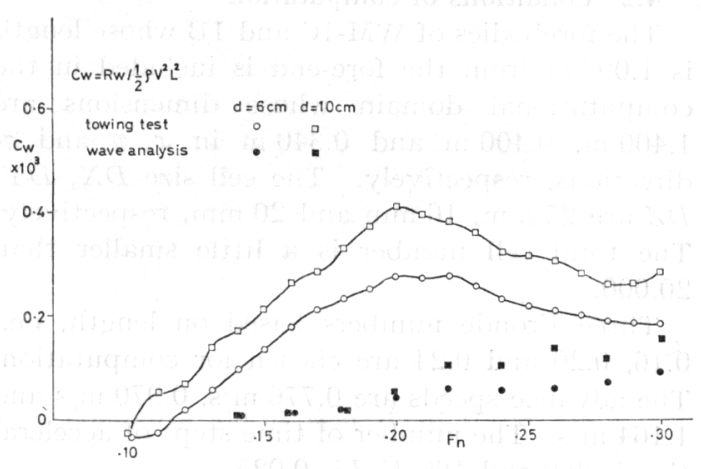

Fig. 8 Wave resistance curves of WM-1B

model WM-1C with an entrance angle of about $9^{\circ}$ generates gentle oblique FSSWs, while WM-1B with a very blunt bow generates two very intense normal FSSWs, one of which is detached from the bow. The appearance is very similar to a turbulent bore in a river. The wave resistance curves derived from towing tests are quite different between each other. It is especially noted that that of WM-1B is far from the wave pattern 
resistance derived from wave analysis in dark marks in Fig. 8 both qualitatively and quantitatively. The FSSWs similar to a bore in Fig. 6 dominantly rule the wave resistance curve of WM-1B which cannot be explained by linear wave resistance theories so far developed. Symbolically saying, the explanation of the wave resistance curve, that has a hump at around $F_{n}=0.20$, is the main purpose of the development of the TUMMAC-II.

It will be better to recall that the FSSW phenomenon has four time-developing stages, i.e., 1) formation of very steep nonlinear waves, 2) breaking of wave crest and energy deficit, 3) diffusion of energy deficit with turbulence and 4) formation of momentum-deficient wake far behind. It is important to note that the stages from 2) to 4) are resultant phenomena that follows 1) and they do not directly influence on wave resistance if higher-order phenomena are ignored. Wave breaking itself does not give resistance to ships, and it is a phenomenon of energy dissipation. Therefore, the hump in the wave resistance of Fig. 8 is due to the generation of nonlinear steep waves called FSSW, that may break.

The present method substantially can cope with the generation of FSSWs immediately before breaking, and can evaluate the FSSW resistance. The shortcoming that breaking is not taken into account does not give serious effect at this preliminary stage of progress.

\subsection{Conditions of computation}

The forebodies of WM-1C and $1 \mathrm{~B}$ whose length is $1.000 \mathrm{~m}$ from the fore-end is included in the computational domain whose dimensions are $1.400 \mathrm{~m}, 0.400 \mathrm{~m}$ and $0.340 \mathrm{~m}$ in $x, y$ and $z$ directions, respectively. The cell size $D X, D Y$, $D Z$ are $25 \mathrm{~mm}, 16 \mathrm{~mm}$ and $20 \mathrm{~mm}$, respectively. The total cell number is a little smaller than 20,000 .

Three Froude numbers based on length, i.e., $0.16,0.20$ and 0.24 are chosen for computation. The advance speeds are $0.776 \mathrm{~m} / \mathrm{s}, 0.970 \mathrm{~m} / \mathrm{s}$ and $1.164 \mathrm{~m} / \mathrm{s}$. The number of time steps of acceleration is 360 and $D T \cdot U / d$ is 0.025 .

The TUMMAC-IIBc method is used in this chapter and the kinematic viscosity of water is assumed zero.

\subsection{Computed results and discussion}

The computed results are shown in Figs. 9 to 14 in three forms of wave contour map, perspective view of wave configuration and pressure coefficient distribution on hull surface.

The difference of the characteristics of bow waves between WM-1C and $1 \mathrm{~B}$ is clearly shown in Figs. 9 to 12, in which wave height is nondimensionalized by $H$. In Figs. 9 and 10, the
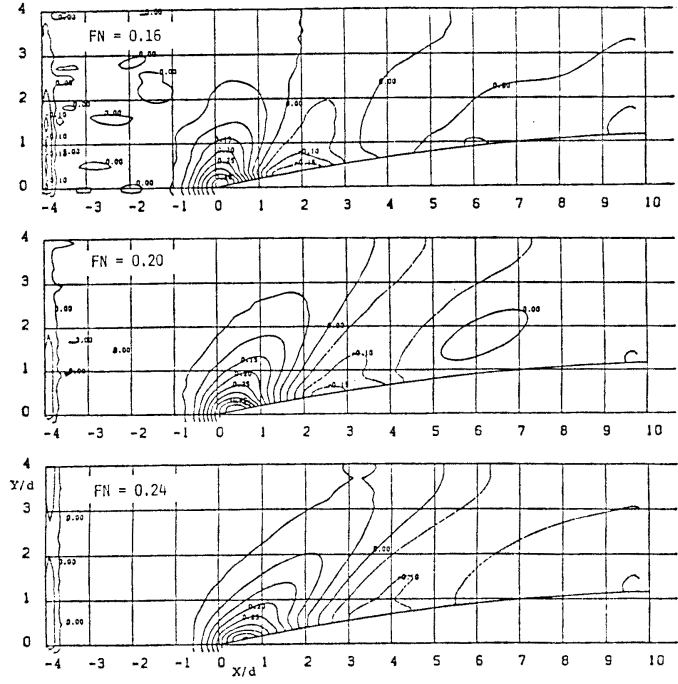

Fig. 9 Computed wave height contours of WM-1C at three Froude numbers
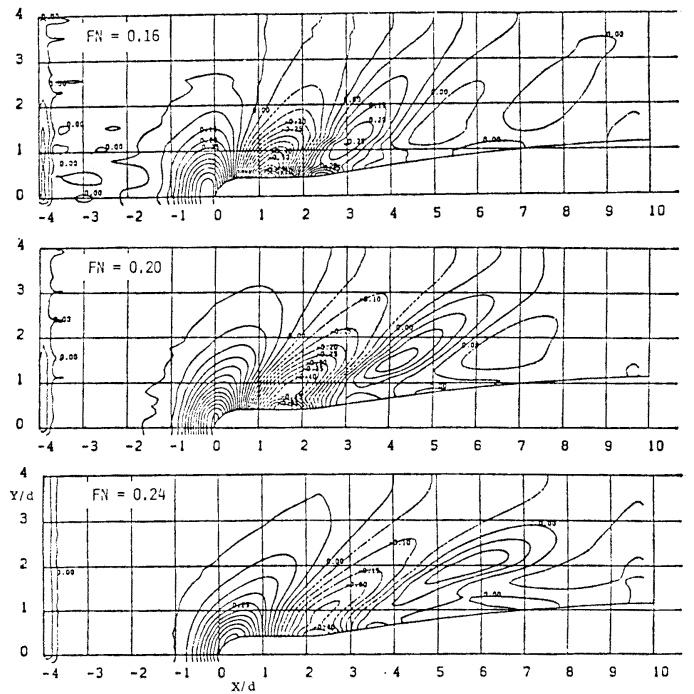

Fig. 10 Computed wave height contours of WM-1B at three Froude numbers

positive and negative contours are drawn in solid and dotted curves, respectively, and the interval of the contours is $0.05 \mathrm{H}$. The waves of WM-1C is rather simple and similar to those of a wedge. The foremost wave is laterally extended and the angle of crest is decreased by the increasing Froude number. Two violent nonlinear waves occur around the forebody of WM$1 \mathrm{~B}$, as expected from Fig. 6 . The foremost wave is always a normal FSSW. Its crest is detached 

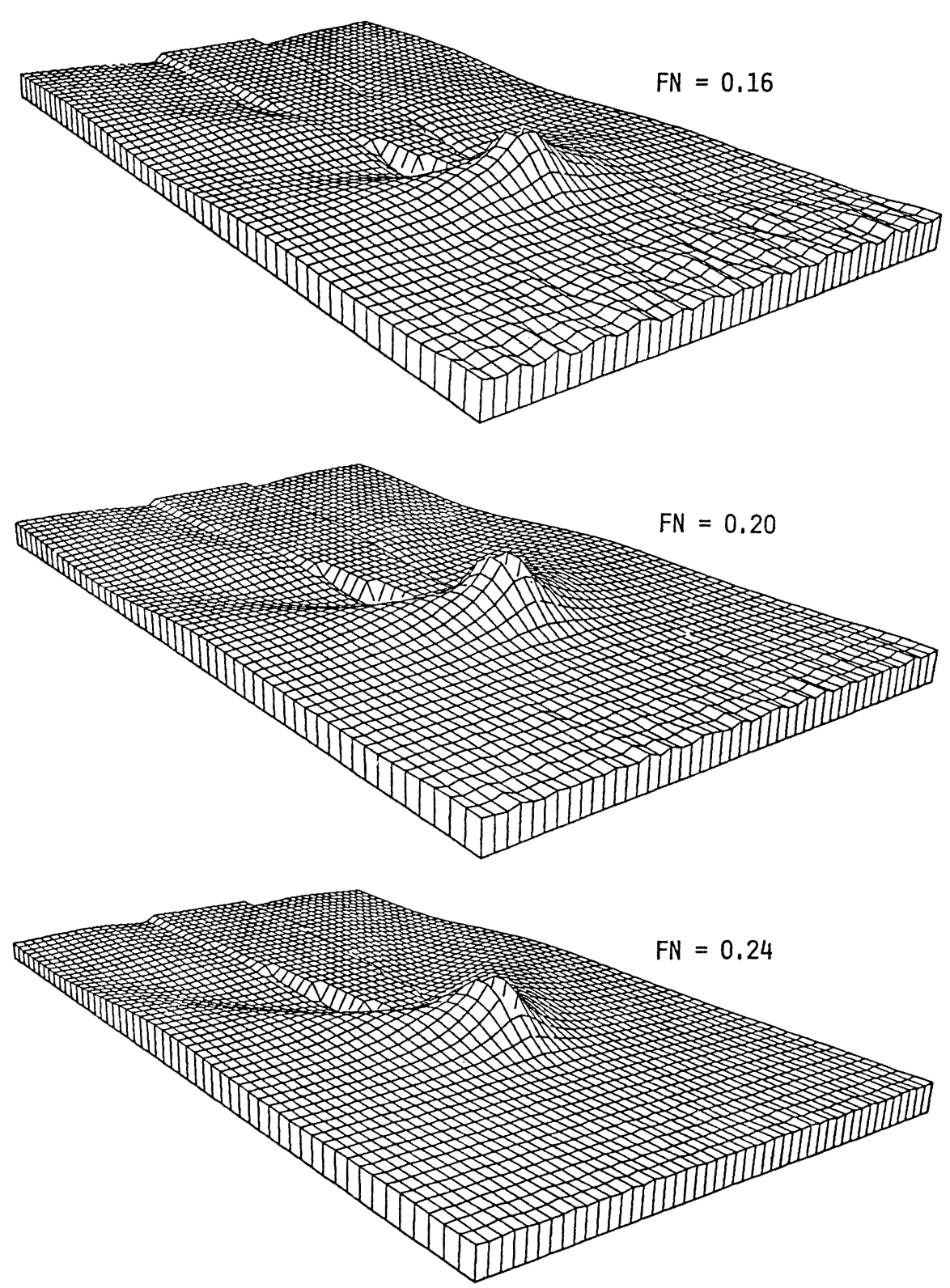

Fig. 11 Computed perspective views of wave configuration of WM-1C at three Froude numbers

from the round bow at low Froude number and it gradually moves backward to be attached. The maximum nondimensional height of the foremost wave is $0.55 H$ at $F_{n}=0.16$, reaches to the highest value of $0.75 \mathrm{H}$ at $F_{n}=0.20$, and then decreases to $0.55 H$ at $F_{n}=0.24$. The second wave starts from the hollow point of the hull surface and its angle of crest is greatly reduced by the increasing Froude number, which is similar to the transition of a normal FSSW to an oblique FSSW. This abrupt change is accompanied by the decrease of the maximum nondimensional wave height, $0.25 H$ of $F_{n}=0.16$ to $0.10 H$ of $F_{n}=0.24$. These changes of FSSW due to increasing Froude number are supposed to be the cause of the wave resistance curve of WM-1B with a hump at $F_{n}=$ 0.20 .

The value that is more intimate with wave resistance is pressure on hull surface as figured in Figs. 13 and 14, in which negative pressure is 

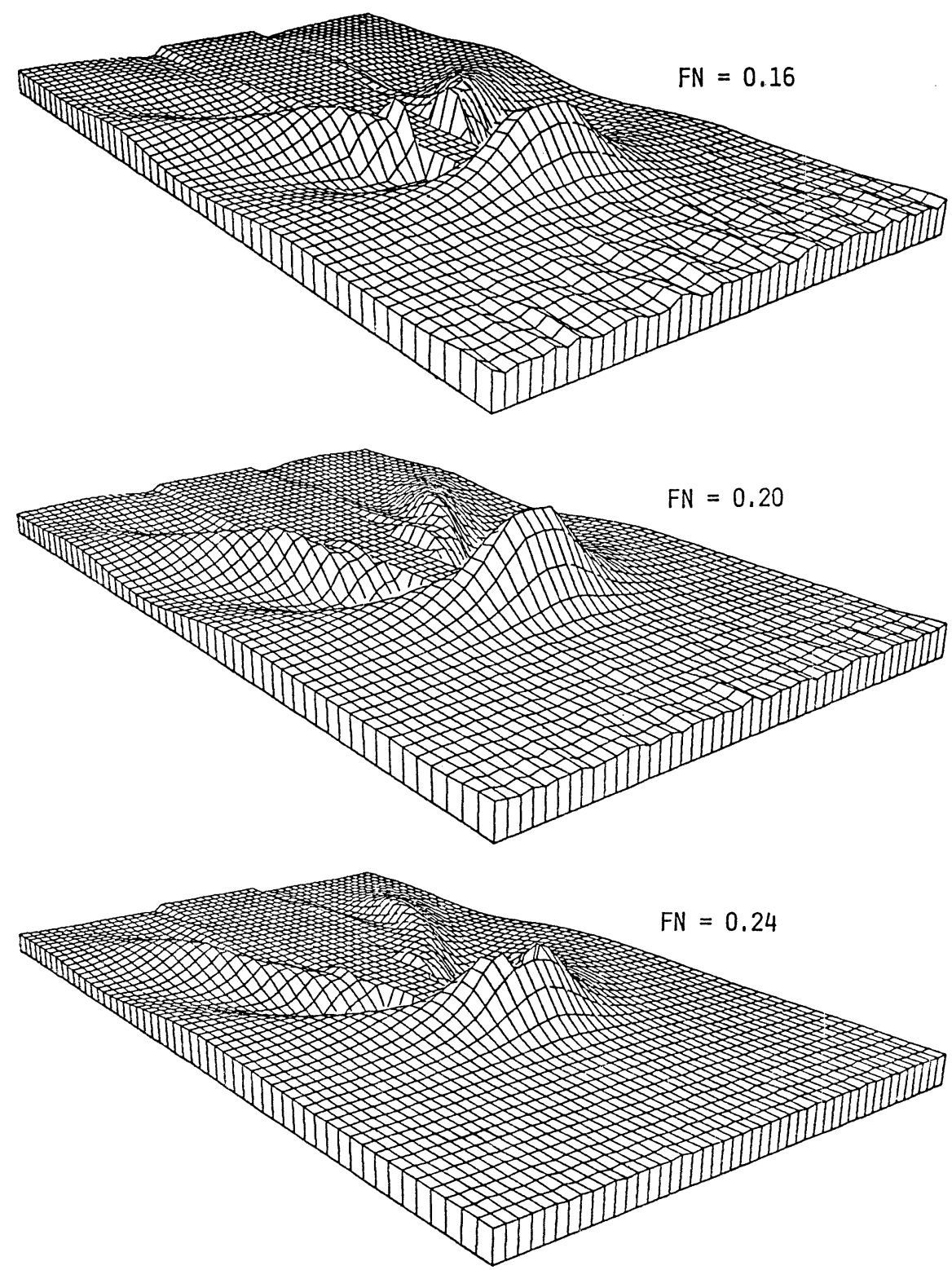

Fig. 12 Computed perspective views of wave configuration of WM-1B at three Froude numbers

indicated by dotted contour curves. The pressure is also nondimensionalized by the head of uniform stream and the undisturbed static head is subtracted, while the wave profiles are dimensional. The close contours near the fore-end, showing high pressure, are supposed to occupy most part of the wave resistance of the forebody. By integrating this contours and taking the $x$ directional component the wave resistance of the forebody is calculated. The obtained values are listed in Table 1. The trends of wave resistance curves in Figs. 7 and 8 are realized, i.e., WMII-1B has a hump around $F_{n}=0.20$, although the wave resistance of a half-body is not measured and the computed results are not directly compared to experiment. For WM-1B both the variation of waves afore-mentioned and the computed resistance predict the hump at $F_{n}=0.20$.

However, the absolute values of WM-1C are too small and they are not reliable. This inadequacy 


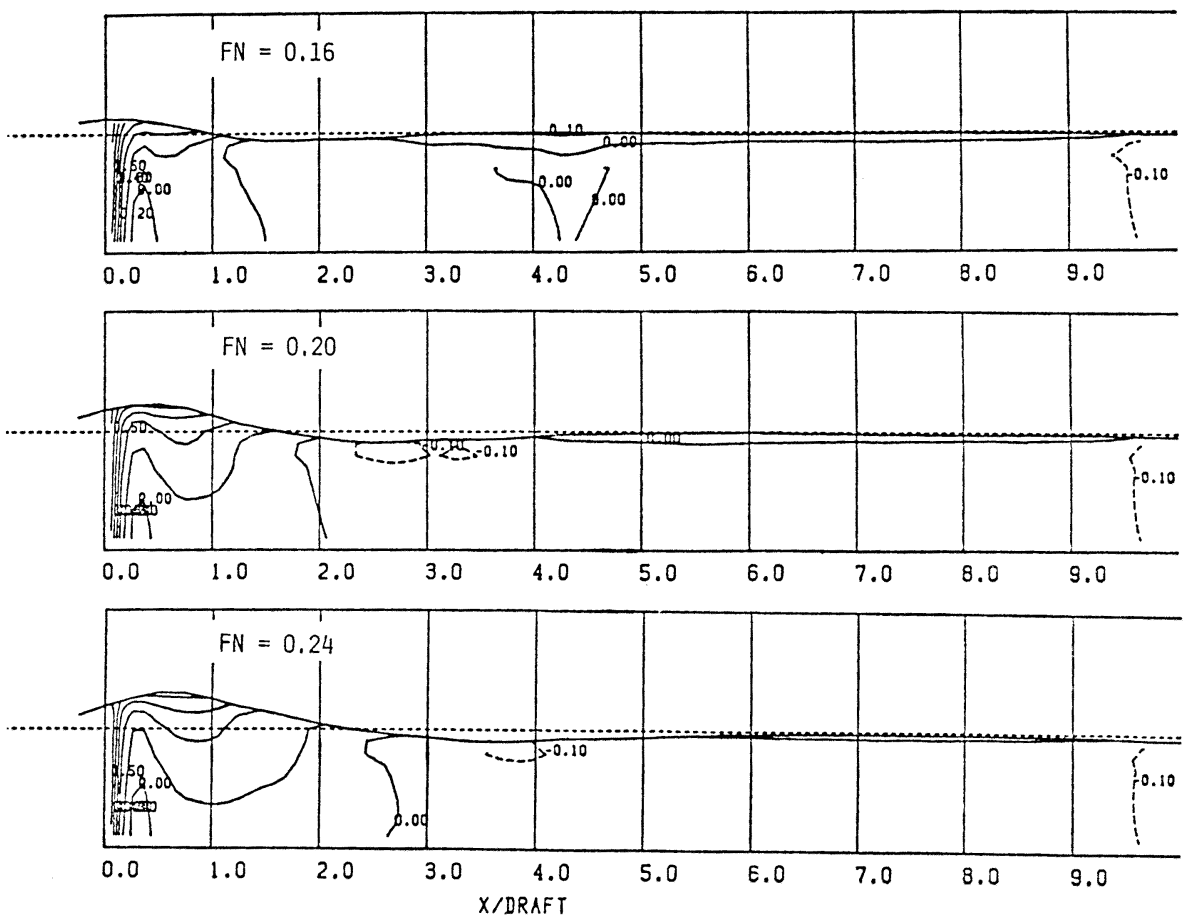

Fig. 13 Computed pressure distribution on the hull surface of WM-1C at three Froude numbers
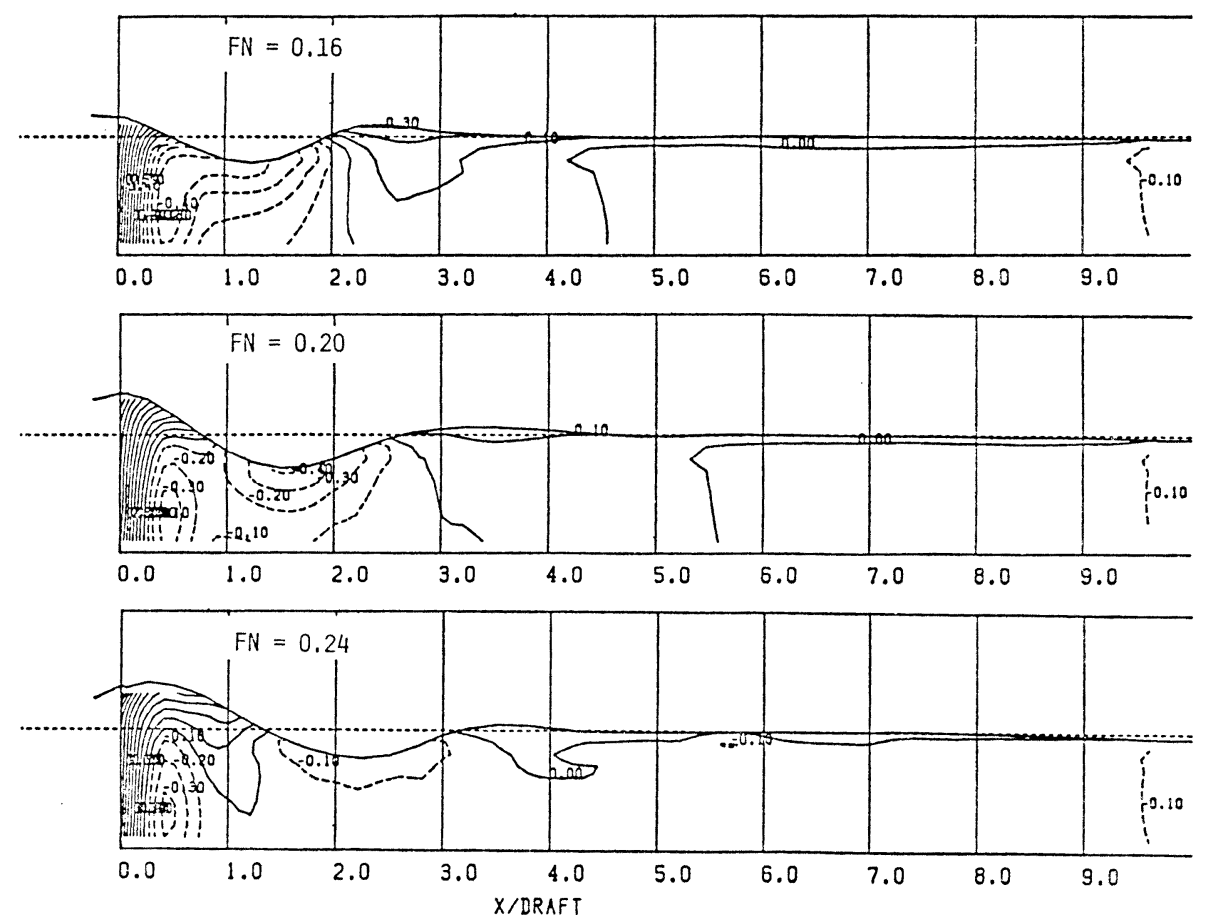

Fig. 14 Computed pressure distribution on the hull surface of WM-1B at three Froude numbers 
Table 1 Computed wave resistance of forebodies

\begin{tabular}{|l|l|l|l|}
\hline Fn & 0.16 & 0.20 & 0.24 \\
\hline WM-1C & $0.035 \times 10^{-3}$ & 0.078 & 0.125 \\
\hline WM-1B & 0.730 & 0.916 & 0.841 \\
\hline
\end{tabular}

$$
\mathrm{C}_{\mathrm{W}}=\mathrm{R}_{\mathrm{W}} / \frac{1}{2} \mathrm{pU}^{2} \mathrm{~L}^{2}
$$

is attributed to the method of pressure integration as well as the degree of accuracy of the TUMMAC-II method. The pressures of boundary cell are used instead of those on the exact location of the hull surface and the integration procedure is not careful enough. These must be improved for the practical purposes.

\section{Concluding remarks}

The number of cell being restricted from economical standpoint, the accuracy of the numerical method was attempted to be improved by applying the concept of the two-step Lax-Wendroff method. This attempt did not resulted in satisfactory completion. But, it showed future possibility of increasing accuracy. The preliminary study on the application of the TUMMAC-II to hull form design showed a fruitful result as well as some points to which further efforts should be devoted. The qualitative accuracy seems to promise us its practical availability in near future.

The computations were carried out by HITAC $\mathrm{M}-200 \mathrm{H} / 280 \mathrm{H}$ at the Computer Center, the University of Tokyo. This research is partly supported by the Grant-in-Aid for Scientific Research of the Ministry of Education, Science and Culture. The authors express cordial thanks to Mr. S. Nishimura and Miss. N. Takiura for their collaboration.

\section{References}

1) Aoki, K. et al: A Numerical Analysis of Nonlinear Waves Generated by Ships of Arbitrary Waterline (First Report), J. Soc. Naval Arch. Japan, Vol. 154 (1983).

2) Burstein, S. Z.: Finite-Difference Calculations for Hydrodynamic Flows Containing Discontinuities, J. Computational Physics 2 (1967).

3) Rubin, E. L. et al: Difference Methods for the Inviscid and Viscous Equations of a Compressible Gas, J. Computational Physics 2 (1967).

4) Inui, $T$. et al: Experimental Investigations on the Wave Making in the NearField of Ships, J. Kansai Soc. Naval Arch., Japan, Vol. 173 (1979).

5) Miyata, H. et al: Characteristics of Free Surface Shock Waves around Wedge Models (Second Report), J. Soc. Naval Arch. Japan, Vol. 151 (1982). 